\title{
Control of Aerodynamic Noise Radiating from Cavity Flows by Introducing Longitudinal Vortices
}

\author{
Hiroshi Yokoyama \\ Department of Mechanical Engineering, Toyohashi University of Technology \\ Toyohashi, Japan \\ h-yokoyama@me.tut.ac.jp
}

\section{Extended Abstract}

Aerodynamic noise is an important issue for high-speed transport vehicles and flow-related machines. Particularly, intense tonal sound often radiates from flows around a cavity, and this sound is referred to as cavity tone.

This cavity tone is due to fluid-acoustic interactions in compressible cavity flows, where the vortex impinging at the downstream wall generates an acoustic wave and the acoustic wave propagating in the upstream direction induces another vortex near the upstream edge. The cavity tone becomes more intense when the spanwise coherence of the vortices becomes higher. Also, the acoustic resonance with standing waves in the cavity tone reinforces the cavity tone.

The control of the cavity tone by introducing streamwise longitudinal vortices into the oncoming boundary layer by plasma actuators (PAs) or blowing jets is focused on in this research. Moreover, the effects of the spanwise pitch of the vortices on the flow and acoustic fields were investigated.

To clarify the effects of the control on the cavity flow, both wind tunnel experiments and direct aeroacoustic simulations were performed. Main experiments and computations were performed at the freestream Mach number of 0.09 , where the oncoming boundary layer is laminar. The ratio of the boundary layer thickness to the cavity length is $\delta / \mathrm{L}=0.055$, where the cavity length is $\mathrm{L}=20 \mathrm{~mm}$. The aspect ratio of the cavity depth to length is $\mathrm{D} / \mathrm{L}=0.5$ and 2.5 , where the acoustic resonance with standing waves occurs at $\mathrm{D} / \mathrm{L}=2.5$. The spanwise pitch of the PA or jets was changed in the range of $\mathrm{p} / \mathrm{L}=0.1$ to 1.25 .

The experiments were performed in a low-noise wind tunnel, and the test section was terminated spanwisely by end walls composed of porous plates. A microphone and hot-wire anemometer were used to measure the sound pressure and velocity. In order to capture the fluid-acoustic interactions in the cavity flow, the simulations are based on the compressible Navier-Stokes equations, where flow and acoustic fields are simultaneously predicted.

The measured results regarding PAs present that the mean reduction level of cavity tone by the PAs with the pitch of $\mathrm{p} / \delta=0.5$ is more than $15 \mathrm{~dB}$ at the freestream velocity of $10-45 \mathrm{~m} / \mathrm{s}$. The predicted results without the control show that the vortices with a spanwisely uniform phase and high coherence are shed in the cavity flow and become the acoustic sources for the cavity tone. The spanwise distributions of the phase and coherence of these vortices become non-uniform and lower by introducing longitudinal vortices by the PAs. Also, the results regarding the control by blowing jets show that longitudinal vortices are introduced into the cavity flow and the cavity tone is reduced. 